\title{
Smoking behavior and psychological dynamics during COVID-19 social distancing and stay-at-home policies: A survey
}

\author{
Pasquale Caponnetto, ${ }^{1-3}$ Lucio Inguscio, ${ }^{4}$ Claudio Saitta, ${ }^{2}$ Marilena Maglia, ${ }^{1,2}$ \\ Francesca Benfatto, ${ }^{1,2}$ Riccardo Polosa ${ }^{1,2}$ \\ ${ }^{1}$ Centro per la Prevenzione e Cura del Tabagismo (CPCT), Azienda Ospedaliero-Universitaria \\ "Policlinico-Vittorio Emanuele", University of Catania; ${ }^{2}$ Center of Excellence for the Acceleration \\ of Harm Reduction (COEHAR), Department of Clinical and Experimental Medicine, University of \\ Catania; ${ }^{3}$ Department of Education, University of Catania; ${ }^{4}$ Department of Psychology, \\ La Sapienza University, Rome, Italy
}

\begin{abstract}
During the COVID-19 pandemic, about 60 million of Italians stayed at home. These circumstances may generate exceptional challenges and stress for people who regularly use cigarettes, ecigarettes, heated tobacco products, dual users, former smokers and never smokers. Here, we present results from a study that was aimed at surveying smoking behaviour and psychological dynamics during the Italian COVID-19 social distancing and stay-at-home
\end{abstract}

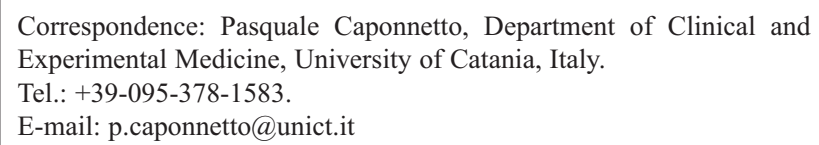

E-mail: p.caponnetto@unict.it

Key words: Smoking; vaping; electronic cigarette; vape shop; heated tobacco products; COVID-19.

Acknowledgements: The authors would like to thank the Italian antismoking League (LIAF) and Dr.ssa Valeria Nicolosi for promoting the survey and encouraging people to participate. No funding was received for this study.

Contributions: The authors contributed equally.

Conflict of interest: See Appendix.

Funding: None.

Availability of data and materials: Study data are available within the text.

Ethics approval and consent to participate: All subjects gave their informed consent for inclusion before they participated in the study conducted in accordance with the Declaration of Helsinki. The study was conducted in accordance with the ethical standards established by the Italian National Psychological Association and approved by the local IERB.

Received for publication: 2 May 2020.

Accepted for publication: 20 May 2020.

This work is licensed under a Creative Commons AttributionNonCommercial 4.0 International License (CC BY-NC 4.0).

(C) Copyright: the Author(s), 2020

Licensee PAGEPress, Italy

Health Psychology Research 2020; 8:9124

doi:10.4081/hpr.2020.9124 policies. Participants living in Italy were invited to complete an online brief questionnaire. A questionnaire was prepared and uploaded in an online survey tool. They were asked to participate regardless of their current smoking status and were divided in seven subgroups. In total, 1825 participants were included in the analysis. Exclusive cigarette smokers; Dual users of cigarette and ecigarettes; Dual users of cigarette and heated tobacco products; Former smokers; Exclusive users of e-cigarette; Exclusive users of heated tobacco products; never smoker. Dual users of cigarette and e-cigarette and exclusive cigarette smokers perceived that their daily consumption has slightly decreased. Exclusive cigarette smokers and exclusive e-cigarette users changed the way of purchasing products. Most exclusive cigarette smokers have considered quitting but most exclusive e-cigarette users have not considered stopping the use of e-cigarettes. In former smokers' group, about one third of participants declared thoughts about starting to smoke again and in never smokers' group few participants declared intention to start smoking. The COVID-19 era could be considered a "transition" phase and as such requires a search for a new balance. These changes in everyday habits can be a significant moment to use established and emerging strategies to create a definitive smoke-free world.

\section{Introduction}

On March 11, 2020, WHO declared Coronavirus (COVID-19) to be a pandemic. Italy was soon one of the most affected countries. From the patient 0 identified on 21 February, the peninsula has recorded new cases daily, which have also been followed by several deaths. To try to contain and manage the spread of the epidemiological emergency, Italy, later followed by many other countries, announced a lock-down. Domestic confinement and the closure of schools, restaurants, sports and recreational activities, shops and many offices were ordered, as well as important limitations in movements and social interactions, causing a huge change in the quality of life of the entire population (Matias et al., 2020). Some recent studies on the psychological and behavioral effects of confinement highlight the emergence in the population of disorders ranging from panic, phobia, health anxiety, sleep disturbances to dissociative symptoms (Banerjee, 2020; Lima et al., 2020; Wang et al., 2020). The psychological effects of confinement seem to increase with each passing day (GarcíaÁlvarez et al., 2020) and in particular this has been found in some groups of the population, such as healthcare personnel (Bai et al., 2004; Maunder et al., 2003), or in vulnerable people with previous 
somatic diseases or with a previous or current mental disorder, including for example depression, anxiety or bipolar disorder (García-Álvarez et al., 2020). Furthermore, it emerges how such isolation measures can have a significant impact on the increased risk of suicide (Reger, Stanley, \& Joiner, 2020). Addicted persons also represent a population at risk and mental health experts report the possible effects of the pandemic on people who suffer or risk developing an addiction (Pfefferbaum \& Nord, 2020; Yao et al., 2020), and among these are those who experience nicotine or cannabinoid addiction. Despite this, individuals who are in a state of domestic isolation frequently continue to use the substances they depend on to relieve stress and negative feelings (Volkow et al., 2020). The use of alcohol, tobacco and other substances can change not only as a form of distraction or behavioral prevention strategy, but also as a result of stress, anxiety or depressive symptoms (García-Álvarez et.al, 2020). For people engaged in a detoxification process, social support is essential, while social isolation is a risk factor because stressful conditions may occur that exacerbate the desire to consume, causing a greater risk of relapse (Kreek et al., 2005). Boredom and free time can also increase the risk of problematic or extreme behavior, such as alcohol consumption and internet addiction (Wang, 2019; Biolcati et al., 2018). A protracted period of isolation, without face-to-face contacts, and a lifestyle based on the activities carried out through the use of technology, encourage the consolidation of negative habits that can lead to video game addictions, compromising mental health, sleep-wake rhythms and/or physical health (King et al., 2020).

All these data show the importance of starting a research to find out how far a change in the consumption model of nicotinecontaining products is taking place (new users, increase/decrease in frequency, relapse, intensity of use, purchase management, intention to quit) as a result of emotional responses, as a moment for change, as an avoidance strategy or an alternative to confinement-related boredom and a pandemic, identifying the responses and strategies developed as an alternative to the aforementioned factors. For these reasons and to better understand how nicotine and no nicotine consumers coped during this crisis, the Center of Excellence for the acceleration of Harm Reduction (CoEHAR) carried out this research with the aim of surveying smoking behaviour and psychological dynamics during the Italian COVID-19 social distancing and stayat-home policies.

\section{Materials and Methods}

A brief questionnaire was prepared by the COEHAR research team and its presentation was uploaded in the Italian antismoking league (LIAF) website (https://www.liaf-italia.it/sondaggio-covid19/) together with informed consents in Italian. If the participant agreed with the informed consent, he/she was redirected to the questionnaire. The informed consent presented the purpose of the survey, the names and contact details of the study investigators, information about who was eligible to take part and how survey data were used, assurances of participant anonymity and confidentiality. Subsequently, participants were asked city of residence, age and gender. No financial or other incentive was offered in exchange for participation and no personal identifying details were collected. The questionnaire was available online for 25 days (from 2 to 26 April 2020). The study was approved by the IERB of the Department of Education Sciences University of Catania. The study was conducted in agreement with the ethical norms set by the Italian National Psychological Association.
The questionnaire was communicated on internet social media and adults were asked to take part, regardless of current or previous smoking status. The IP address of the participants was recorded in order to avoid double entries. Previous and contemporary smoking status was asked and, based on the latter, participants were divided into seven groups for the analysis: Exclusive cigarette smokers (packaged or rolled); Dual users of cigarette and electronic cigarette; Dual users of cigarette and heated tobacco products (e.g. IQOS or GLO); Former smokers (had smoked in the past but not now); Exclusive users of electronic cigarette; Exclusive users of heated tobacco products (e.g. IQOS or GLO); Never smokers. The questionnaire included questions about how had this stressful situation impacted access and product use behavior among smokers, vapers, dual users, former smokers and never smokers.

To assess changes in consumption, the following questions were asked: "During this period of forced confinement due to the coronavirus emergency, do you believe that you have increased or decreased the number of cigarettes smoked per day?"; "During this period of forced confinement due to the coronavirus emergency, do you believe that you have increased or decreased the number of liquid/cartridges?"; "During this period of forced confinement due to the coronavirus emergency, do you believe that you have increased or decreased the number of heets/sticks?". The answers were scored as: 1, "Increased"; 2, "Decreased"; 3, "Stable".

To examine the significant changes in the way of purchasing, the following questions were asked: "During this period of forced confinement due to the coronavirus emergency, how are you managing to purchase cigarettes?"; "During this period of forced confinement due to the coronavirus emergency, how are you managing to purchase e-liquid/cartridges?"; "During this period of forced confinement due to the coronavirus emergency, how are you managing to purchase heets/sticks?"; The answers were scored as: 1, "As always 2, "It has changed (Please, describe how your way of buying cigarettes has changed)".

To examine the thoughts about quitting, the following questions were asked: "During this period of forced confinement due to the coronavirus emergency, have you thought about quitting smoking or possible alternatives such as switching to the exclusive use of electronic cigarettes or the exclusive use of heated tobacco products (e.g. IQOS or GLO)?; "During this period of forced confinement due to the coronavirus emergency, have you thought about stopping using the electronic cigarette?"; "During this period of forced confinement due to the coronavirus emergency, have you thought about stopping using the heated tobacco products?"; The answer were scored as: 1, "No"; 2, "Yes".

To examine the risk of relapse among former smokers and the risk of starting smoking in those who had never smoked, the following questions were asked: "During this period of forced confinement due to the coronavirus emergency, have you thought about starting to smoke again?"; "During the COVID-19 restriction period, did you think about starting to smoke?"; The answers for former smokers were scored as: 1, "No"; 2, "Yes but I haven't started smoking conventional cigarettes again"; 3, "Yes and I started smoking conventional cigarettes again"; The answers for never smokers were scored as: 1, "No"; 2, "Yes I thought about starting to smoke cigarettes"; 3, "Yes I thought about starting to smoke electronic cigarettes"; 4, "Yes I thought about starting to smoke heated tobacco cigarettes (e.g. IQOS or GLO)"

\section{Statistical analysis}

Participants were categorized according to their reported smoking status at the time of participation to the survey. Results were reported for the whole sample and for each of the subgroups. 
The sample size varied by variable because of missing data. Kolmogorov-Smirnoff tests were performed to assess normality of distribution of variables. Categorical variables are reported as number (percentage). Cross tabulations with $\chi 2$ test and residual standardized values were used for categorical variables. A two-tailed $P$ value of considered statistically significant, and all analyses were performed with commercially available statistical software (SPSS v. 25, Chicago, IL, USA).

\section{Results}

\section{Participants characteristics}

1825 participants were included into the analysis and characteristics of the study group are presented in Table 1. The average age was 34.7 years (sd: 14.11), with female and young adult predominance. The vast majority were exclusive cigarette smoker $(32 \%)$ or never smoker $(30 \%)$, with a small proportion of dual user of cigarette and heated tobacco products $(1.8 \%)$ or dual user of cigarette and electronic cigarette (3.5\%). Former smokers were $16 \%$ and finally, exclusive user of heated tobacco products and exclusive user of electronic cigarette were respectively $4.4 \%$ and $12.3 \%$.

\section{Changes in consumption}

A chi-square test of independence showed that there was a significant association between "users group" variable and categorial variable "During this period of forced confinement due to the coronavirus emergency, do you believe that you have increased or decreased the consumption of [specific product]", $X^{2}$ $(4, \mathrm{~N}=985)=33.17, \mathrm{p}=.001$. Considering the standardized residual values (sr), the "Dual users of combustible tobacco and Ecig" and "Exclusive combustible tobacco users" reported a negative sr value (-2.7 and -1.9) for "Stable" category; also "Exclusive Ecig users" reported a sr positive value of 4.9. For the "Down" category, we found a positive sr value of 2.9 for "Exclusive combustible tobacco users" and a negative sr value (-5.7) for the "Exclusive Ecig users". "Dual users of combustible tobacco and Ecig" and "Exclusive combustible tobacco users" slightly decreased the number of cigarettes or e-liquid and in comparison, to percentage values the "Exclusive Ecig users" had a stable level of consumption (Table 2 and Figure 1).

\section{Changes in the way of purchasing}

A chi-square test of independence showed that there was a significant association between "users group" variable and categorial variable "During this period of forced confinement due to the coronavirus emergency, how are you managing to purchase [specific product]", $X^{2}(4, \mathrm{~N}=985)=33.17, \mathrm{p}=.001$. Considering the standardized residual values, $(\mathrm{sr}<1.96$ and $\mathrm{sr}<-1.96)$, the "Exclusive combustible tobacco user" and "Exclusive Ecig users" group reported a $\mathrm{sr}=-2.0$ and $\mathrm{sr}=-3.2$, for response category "As always" and positive values for category "It has changed" $\mathrm{sr}=2.3$ and $\mathrm{sr}=-3.6$. During the lockdown period, the "Exclusive combustible tobacco users" and the "Exclusive Ecig users" changed the way of purchasing products and the majority of them changed their habits by having large stocks of cigarettes or eliquids/cartridges at home in order to avoid going out every day (Table 3 and Figure 2).

\section{Thoughts about quitting}

A chi-square test of independence showed that there was a significant association between "users group" variable and categorial variable "During this period of forced confinement due to the coronavirus emergency, have you thought about quitting smoking or possible alternatives such as switching to the exclusive use of electronic cigarettes or the exclusive use of heated tobacco products [specific product]", $X^{2}(6, \mathrm{~N}=1825)=305.5, \mathrm{p}=.001$. Considering the standardized residual values, for the "No" level of

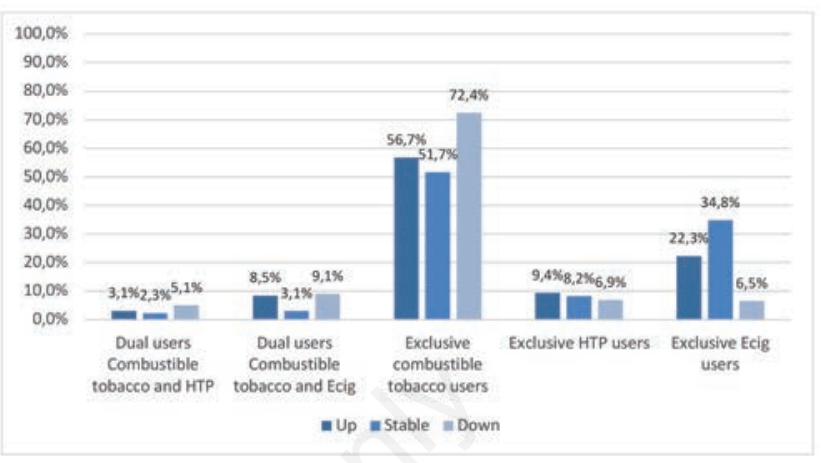

Figure 1. Changes in consumption.

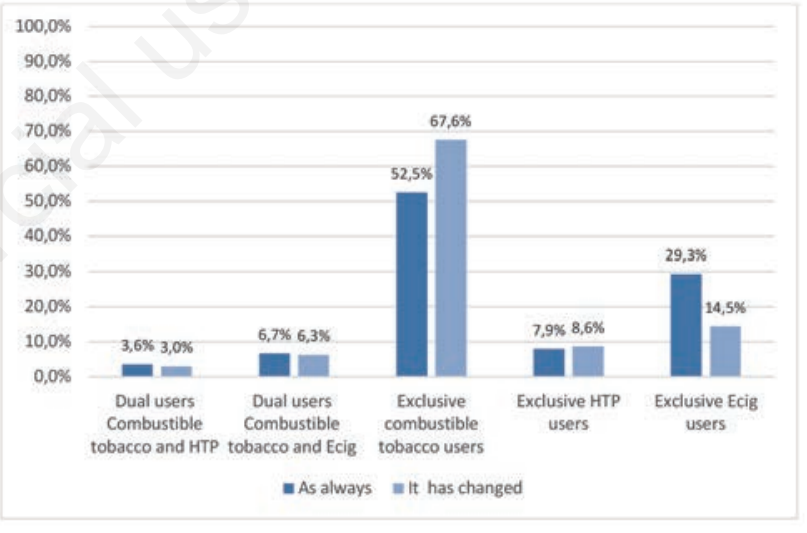

Figure 2. Changes in the way of purchasing.

Table 1. Characteristics of the study participants.

\begin{tabular}{lcc} 
& n & $\%$ \\
Gender & 683 & 37.5 \\
Men & 1142 & 62.5 \\
Woman & 1825 & 100 \\
Age group & & \\
18-28 & 889 & 48.7 \\
$29-39$ & 368 & 20,1 \\
$40-50$ & 257 & 14,1 \\
$51-61$ & 237 & 13 \\
$62+$ & 74 & 4,1 \\
\hline Group & & \\
Dual user of cigarette and heated tobacco products & 33 & 1,8 \\
Dual user of cigarette and electronic cigarette & 64 & 3,5 \\
Former smoker & 293 & 16 \\
Exclusive cigarette smoker & 582 & 32 \\
Never smoker & 547 & 30 \\
Exclusive user of heated tobacco products & 81 & 4,4 \\
Exclusive user of electronic cigarette & 225 & 12,3 \\
\hline
\end{tabular}


categorial variable, "Exclusive combustible tobacco users" and "Exclusive Ecig users" reported a significant value; the sr value is negative ( $\mathrm{sr}=-6.2$ ) for "Exclusive combustible tobacco user" group with positive values (4.9 and 2.3) and for "Exclusive Ecig users". For the "Yes" level, the sr value is positive ( $\mathrm{sr}=11.6)$ for "Exclusive combustible tobacco users" and negative value $(\mathrm{sr}=-4.2)$ in "Exclusive Ecig users". Significantly, exclusive combustible tobacco users were the ones who would mostly have liked to quit, but those who smoke exclusively e-cigarettes were the ones who didn't want to quit (Table 4 and Figure 3).

\section{Risk of relapse for former smokers and the risk of starting smoking for never smokers}

In the "former smokers" group, we compared percentage value of response (Yes/No) to item "During this period of forced confinement due to the coronavirus emergency, have you thought about starting to smoke again?" and the participants showed a percentage value of "Yes, I thought of starting" of $29.7 \%$ (Figure 4). Finally, in never smokers' group we found that almost $2,3 \%$ of participants said they would start smoking by using cigarettes, $0.8 \%$ e-cigarette, $0 \%$ Heated Tobacco Product (HTP) and 97\% said they would not start smoking (Figure 5).

\section{Discussion}

This is the first survey that specifically focused on the psychological dynamics of smoking behavior during Italian COVID-19 social distancing and stay-at-home policies. A large number of smokers, never smokers, former smokers, vapers and dual users participated; This study focused on a careful observation of the types of tobacco-related products (cigarette, e-cigarette, heated tobacco and dual usage) inserted within the italian lockdown period. Within this time frame, it could be of interest to observe such behaviour under a "psychological lens" that explains how and how much cigarette dependence or attachment to a low-risk product has affected the study participants' behaviour (Baltes et al., 2009). The first data to be observed is that of the declared slight decrease in the

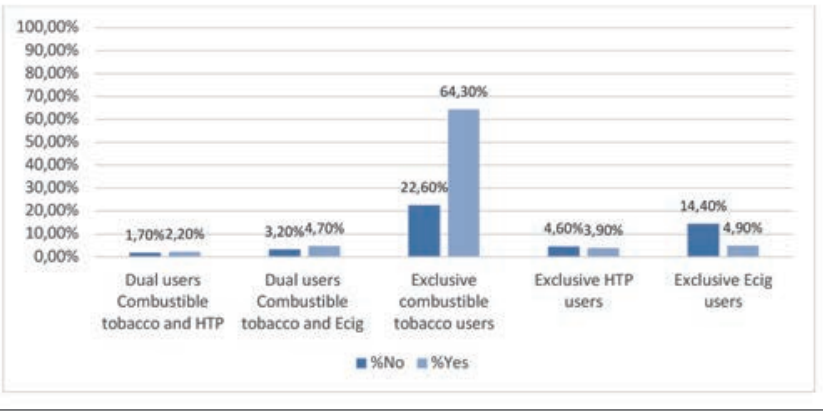

Figure 3. Thoughts about quitting.

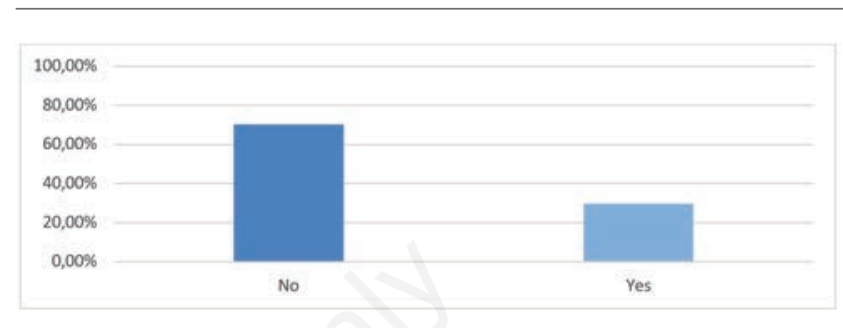

Figure 4. Risk of relapse for former smokers.

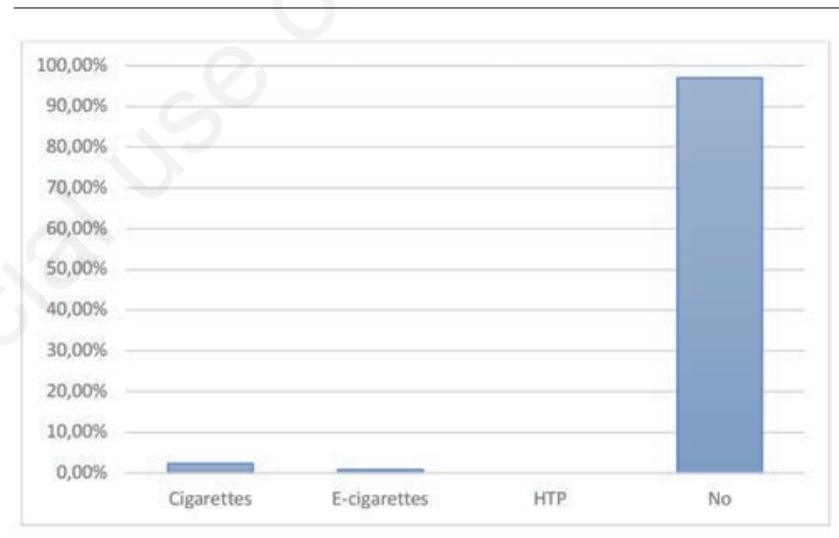

Figure 5. risk of starting smoking for never smokers.

Table 2. Changes in consumption.

\begin{tabular}{lccccc} 
& $\begin{array}{c}\text { Dual user Combustible } \\
\text { tobacco and HrP }\end{array}$ & $\begin{array}{c}\text { Dual user Combustible Exclusive combustible } \\
\text { tobacco and Ecig }\end{array}$ & $\begin{array}{c}\text { Exclusive HitP users } \\
\text { tobacco user }\end{array}$ & Exclusive Ecig users \\
Up & $3,1 \%(\mathrm{sr}=-0.2)$ & $8,5 \%(\mathrm{sr}=1.4)$ & $56,7 \%(\mathrm{sr}=-0.5)$ & $9,4 \%(\mathrm{sr}=0.7)$ & $22,3 \%(\mathrm{sr}=-0.2)$ \\
Stable & $2,3 \%(\mathrm{sr}=-1.1)$ & $3,1 \%(\mathrm{sr}=-2.7)$ & $51,7 \%(\mathrm{sr}=-1.9)$ & $8,2 \%(\mathrm{sr}=0$ & $34,8 \%(\mathrm{sr}=4.9)$ \\
\hline Down & $5,1 \%(\mathrm{sr}=1.6)$ & $9,1 \%(\mathrm{sr}=1.7)$ & $72,4 \%(\mathrm{sr}=2.9)$ & $6,9 \%(\mathrm{sr}=-0.8)$ & $6,5 \%(\mathrm{sr}=-5.7)$ \\
\hline
\end{tabular}

Table 3. Changes in the way of purchasing.

\begin{tabular}{|c|c|c|c|c|c|}
\hline & $\begin{array}{l}\text { tual user Combustible } \\
\text { tobacco and HTP }\end{array}$ & $\begin{array}{c}\text { Dual user Combustible } \\
\text { tobacco and Ecig }\end{array}$ & Exclusive combustible & $\begin{array}{l}\text { Exclusive HTP users } \\
\text { tobacco user }\end{array}$ & Exclusive Ecig users \\
\hline As always & $3,6 \%(\mathrm{sr}=0.3)$ & $6,7 \%(\mathrm{sr}=0.1)$ & $52,5 \%(\mathrm{sr}=-2.0)$ & $7,9 \%(\mathrm{sr}=-0.3)$. & $29,3 \%(\mathrm{sr}=3.2)$ \\
\hline It has changed & d $\quad 3,0 \%(\mathrm{sr}=-0.4)$ & $6,3 \%(\mathrm{sr}=-0.2)$ & $67,6 \%(\mathrm{sr}=2.3)$ & $8,6 \%(\mathrm{sr}=0.3)$ & $14,5 \%(\mathrm{sr}=-3.6)$ \\
\hline
\end{tabular}

Table 4. Thoughts about quitting.

\begin{tabular}{|c|c|c|c|c|c|}
\hline & $\begin{array}{l}\text { Dual user Combustible } \\
\text { tobacco and HTP }\end{array}$ & $\begin{array}{c}\text { Dual user Combustible } \\
\text { tobacco and Ecig }\end{array}$ & Exclusive combustible & $\begin{array}{c}\text { Exclusive HTP users } \\
\text { tobacco user }\end{array}$ & Exclusive Ecig users \\
\hline No & $1,70 \%(\mathrm{sr}=-0.3)$ & $3,20 \%(\mathrm{sr}=-0.7)$ & $22,60 \%(\mathrm{sr}=-6.2)$ & $4,60 \%(\mathrm{sr}=0.3)$ & $14,40 \%(\mathrm{sr}=2.3)$ \\
\hline Yes & $2,20 \%(\mathrm{sr}=-0.6)$ & $4,70 \%(\mathrm{sr}=1.3)$ & $64,30 \%(\mathrm{sr}=11.6)$ & $3,90 \%(\mathrm{sr}=-0.5)$ & $4,90 \%(\mathrm{sr}=-4.2)$ \\
\hline
\end{tabular}


consumption of cigarettes during the lockdown period. This aspect may be linked to several factors including: 1) the lack of opportunities for smokers to live the usual habits related to the consumption of cigarettes such as the cigarettes smoked during the Italian ritual of drinking coffee at a bar or while driving home/work or home/university and given that the majority of the participants were young, it is likely that the perceived reduction in cigarette smoking could be also attributable to the absence of daily socialization in places of youth gathering (Mucci et al., 2020). If such habits that accurately marked the rhythms of the smoker's life were missed consequently an unintentional decrease in cigarette consumption could be observed; 2) Another aspect to note is that of the variation of the family lifestyle habits of each smoker; for example, spending more time in the home with children or nonsmoking cohabiting partners has likely led people to slightly reduce their consumption of cigarettes in order to respect their family members (Thomer et al., 2019); 3) fear of COVID-19 development as coughing is common in both smoking and COVID-19 positives (Patanavanich \& Glantz, 2020). However, if this data can only be linked to traditional cigarette smokers, the same cannot be said for those who use e-cigarettes, this category is not similarly linked to certain "behavioral" patterns typical of cigarette smokers, so within this survey there is a trend indicating a slight increase in daily consumption among vapers during the lock-down period (Adriens et al., 2018). The second data to be observed is that of how products are purchased. The data provided showed that there has been an increase in the purchase of products such as cigarettes and e-liquids. The response to this behaviour is dictated by the fact that during the lockdown most consumers of smoking products have likely experienced states of anxiety and concern related to the possible "lack" of something considered "fundamental" (Lee, 2020). The anxieties related to the concern of being without basic necessities has affected not only smokers but the entire population (Brooks et al., 2020). The supply of consumer goods for smokers can be comparable to that of all citizens who poured outside every supermarket trying in every way to stock up products such as yeast or flour, the "must" of quarantine. This element emerges from a recent study that confirms how citizens around the world have behaved in a "protective" way dictated by a sudden and most violent sense of frustration, sense of marked loneliness and the concern related to the fear of unavailability of essential products, each of these sensations accompanied by a strong discomfort linked to the constant fear of contagion (Troisi, 2020). Our sample is limited to the Italian population and we observed a female and a young predominance in participation to this survey. Further studies are needed to explore this phenomenon and define whether our study findings will be confirmed or not. The survey was announced and promoted in popular websites. Therefore, it was expected that young users able to use technology and social network with a not eradicate cigarette dependence would mainly participate, and the high proportion of young smokers and never smokers confirms this. However, it is important to consider that having information on smoking behaviour of young people is important given that they represent the future of our society, since this can provide health officials with information on how to disseminate health empowerment messages, especially during this sad period. Therefore, it would be realistic and valuable to create and disseminate innovative and effective way of sustaining a healthy lifestyle by promoting smoking cessation for smokers, avoiding smoking initiation for never smokers and relapse for former smokers and to properly inform the public that the moment of COVID-19 is creating a land of psychological change where to sow seeds of health.

\section{Conclusions}

The COVID-19 era will be looked back into history as an era characterized by great upheaval that has upset every human rhythm and has not been experienced as a dive into the unknown, a feeling that was replicated day after day. It can and should be considered a "transition" phase and as such requires a search for a new balance. What is certainly most encouraging from reading the research data is that those who have quit smoking before COVID-19 have not relapsed thanks to their personal resilience and coping skills. The former smokers interviewed found their comfort zone in the home during this period, far from confrontation with others, thus not allowing the onset of psychological suffering related to the lack of cigarettes. These changes in everyday habits can be a significant moment to use established and emerging strategies to create a definitive smoke-free world.

\section{References}

Adriaens, K., Van Gucht, D., Baeyens, F. (2018). Differences between Dual Users and Switchers Center around Vaping Behavior and Its Experiences Rather than Beliefs and Attitudes. International Journal of Environmental Research and Public Health, 15, 12.

Bai, Y., Lin, C.C., Lin, C.Y., Chen, J.Y., Chue, C.M., \& Chou, P. (2004). Survey of stress reactions among health care workers involved with the SARS outbreak. Psychiatric Services, 55(9), 1055-1057. doi:10.1176/appi.ps.55.9.1055

Baltes, B.B. (2009). PSYCHOLOGICAL CLIMATE: A COMPARISON OF ORGANIZATIONAL AND INDIVIDUAL LEVEL REFERENTS. Human Relations, 62(5): 669-700.

Banerjee, D. (2020). The COVID-19 outbreak: Crucial role the psychiatrists can play. Asian journal of psychiatry, 50, 102014. doi: 10.1016/j.ajp.2020.102014.

Biolcati, R., Mancini, G., \& Trombini, E. (2018). Proneness to boredom and risk behaviors during adolescents' free time. Psychological reports, 121(2), 303-323.

Brooks, S.K., Webster, R.K., Smith, L.E., Woodland, L., Wessely, S., Greenberg, N. and Rubin, G.J., 2020. The psychological impact of quarantine and how to reduce it: rapid review of the evidence. The Lancet.

García-Álvarez, L., de la Fuente-Tomás, L., Sáiz, P. A., GarcíaPortilla, M. P., \& Bobes, J. (2020). Se observarán cambios en el consumo de alcohol y tabaco durante el confinamiento por COVID-19 Adicciones, 32(2), 85-89.

King, D. L., Delfabbro, P. H., Billieux, J., \& Potenza, M. N. (2020). Problematic online gaming and the COVID-19 pandemic. Journal of Behavioral Addictions.

Lee SA (2020). Coronavirus anxiety scale: A brief mental health screener for covid-19 related anxiety. Death Studies. April 2020. doi:10.1080/07481187.2020.1748481.

Matias T, Dominski FH, Marks DF. (2020). Human needs in COVID-19 isolation. $J$ Health Psychol. 2020 May 6:1359105320925149. doi: 10.1177/1359105320925149. [Epub ahead of print]

Maunder, R., Hunter, J., Vincent, L., Bennett, J., Peladeau, N., Leszcz, M., \& Mazzulli, T. (2003). The immediate psychological and occupational impact of the 2003 SARS outbreak in a teaching hospital. Canadian Medical Association Journal, 168(10), 1245-1251.

Mucci F, Mucci N, Diolaiuti F (2020). Lockdown and isolation: 
Psychological aspects of COVID-19 pandemic in the general population. Clinical Neuropsychiatry: Journal of Treatment Evaluation, 17(2):63-64.

Patanavanich R, Glantz SA (2020). Smoking is Associated with COVID-19 Progression: A Meta-Analysis. Nicotine Tobacco Research. pii: ntaa082. doi: 10.1093/ntr/ntaa082. [Epub ahead of print]

Pfefferbaum, B., \& North, C.S. (2020). Mental health and the Covid-19 pandemic. New England Journal of Medicine.

Reger, M. A., Stanley, I. H. y Joiner, T. E. (2020). Suicide mortality and coronavirus disease 2019-A perfect storm? JAMA Psychiatry. doi:10.1001/jamapsychiatry.2020.1060.

Thomeer MB, Hernandez E, Umberson D, Thomas PA (2019). Influence of Social Connections on Smoking Behavior across the Life Course. Advances in Life Course Research.

Troisi A (2020). Fear of COVID-19: Insights from evolutionary behavioral science. Clinical Neuropsychiatry: Journal of Treatment Evaluation, 17(2), 72-75

Volkow, N. D. (2020). Collision of the COVID-19 and addiction epidemics. Annals of Internal Medicine.

Yao, H., Chen, J. H., \& Xu, Y. F. (2020). Patients with mental health disorders in the COVID-19 epidemic. The Lancet Psychiatry, 7(4), e21.

Wang, C., Pan, R., Wan, X., Tan, Y., Xu, L., Ho, C. S. y Ho, R. C. (2020). Immediate psychological responses and associated factors during the initial stage of the 2019 coronavirus disease (COVID-19) epidemic among the general population in China. International Journal of Environmental Research and Public Health, 17(5). doi:10.3390/ijerph17051729

Wang, W. C. (2019). Exploring the relationship among free-time management, leisure boredom, and internet addiction in undergraduates in Taiwan. Psychological reports, 122(5), 1651-1665. 